\title{
INVARIANTS OF THE RATIONAL PLANE QUINTIC CURVE AND OF ANY RATIONAL CURVE OF ODD ORDER*
}

BY

\author{
JOSEPH EUGENE ROWE
}

1. Introduction. The subject of Rational Curves is a growing one, and it is desirable to have in our mathematical literature a maximum of information in regard to them in a minimum of printed pages. Invariants of rational curves are rather long expressions and instead of encumbering a journal with such expressions it is much better to use if possible some expression which already occurs in a convenient place. In my dissertation, a piece of work done under Dr. Morley's direction, I have shown among other things how certain combinants of two binary quartics, explicitly written out in Salmon, $\uparrow$ may be interpreted as invariants of the rational plane quartic which I call the $R^{4}$. In this paper I am going to develop a method by which these same combinants may be interpreted as invariants of any rational plane curve of odd order, and the process will be carried out in detail for the $R^{5}$ as an illustrative example and because of the special interest which invariants of the plane $R^{5}$ possess.

2. Transvectants of Line Sections. Let the parametric equations of a rational plane curve of order $n$, which I shall refer to as the $R^{n}$, be

$$
x_{i}=\left(\alpha_{i} t\right)^{n}
$$

$(i=0,1,2)$,

in symbolic expressions which are the equivalent of

$$
x_{i}=a_{i} t^{n}+C_{1}^{n} b_{i} t^{n-1}+C_{2}^{n} c_{i} t^{n-2} \cdots \quad(i=0,1,2) .
$$

If (1) is cut by the two lines

$$
(\xi x)=\xi_{0} x_{0}+\xi_{1} x_{1}+\xi_{2} x_{2}=0,
$$

$$
(\eta x)=\eta_{0} x_{0}+\eta_{1} x_{1}+\eta_{2} x_{2}=0,
$$

we obtain two binary $n$-ics which may be written symbolically

$$
\begin{aligned}
& (\beta t)^{n}=0, \\
& (\gamma t)^{n}=0 .
\end{aligned}
$$

* Presented to the Society, April 25, 1914.

$\dagger$ Higher Algebra, Fourth Edition, pp. 219-222. 
The expression

$$
(\beta \gamma)^{r}(\beta t)^{n-r}(\gamma t)^{n-r}=0
$$

is a function of (5) and (6) and is called the $r$ th transvectant of (5) and (6). If $x_{0}, x_{1}, x_{2}$ are substituted for the coördinates of the point in which (3) and (4) intersect, these transvectants may be expressed as binary forms in $t$ of degree $2(n-r)$ whose coefficients are linear functions of the three-rowed determinants of the type

$$
\left|\begin{array}{lll}
a_{0} & b_{0} & x_{0} \\
a_{1} & b_{1} & x_{1} \\
a_{2} & b_{2} & x_{2}
\end{array}\right| \equiv|a b x|,
$$

so long as $r$ is an odd number. For instance, if $r=1$, (7) becomes the tangential equation of the $R^{n}$, that is, it becomes a binary form equated to zero in which, if a particular value of $t$, say $t_{1}$, is substituted, the result is the equation of the tangent to the $R^{n}$ at the point whose parameter is $t_{1}$; but if the coördinates of a particular point are inserted for $x_{0}, x_{1}, x_{2}$, the resulting binary form equated to zero has $2(n-1)$ roots which are the parameters of the $2(n-1)$ tangents to the $R^{n}$ through this particular point.

In particular, if $r=n$ in (7), that expression becomes

$$
(\beta \gamma)^{n}=0 .
$$

For any odd value of $n$ this becomes, by means of the scheme described above, the equation of $a$ covariant line of the corresponding $R^{n}$, and it will be referred to as the covariant line of the $R^{n}$ since it has the distinction of being its simplest covariant line. An idea of the appearance of the equations of these lines may be formed by carrying out in detail the process just indicated for a few of the $R^{n}$. I give several below, indicating the particular $R^{n}$ and the corresponding covariant line $L_{n}$.

$$
\begin{gathered}
R^{3} \text { has } L_{i} \equiv|a d x|-3|b c x|=0 . \\
R^{5} \text { has } L_{5} \equiv|a f x|-5|b c x|+10|c d x|=0 . \\
R^{7} \text { has } L_{7} \equiv|a b x|-7|b g x|+21|c f x|-35|d e x|=0 .
\end{gathered}
$$

The equations of others may be written out from analogy with these.

3 . Osculants of $R^{n}$. The equations

$$
x_{i}=\left(a_{i} t^{\prime}\right)^{s}\left(\alpha_{i} t\right)^{n-s} \quad(i=0,1,2),
$$

where $t^{\prime}$ is constant, define a rational curve of order $n-s$ which is called the $s$ th osculant of the $R^{n}$ at $t^{\prime}$, or the osculant $(n-s)$-ic of the $R^{n}$ at $t^{\prime}$. Its parametric equations are, of course, simply (13). For instance, the osculant 
conic of the $R^{3}$ at $t^{\prime}$ is

$$
x_{i}=\left(a_{i} t^{\prime}+b_{i}\right) t^{2}+2\left(b_{i} t^{\prime}+c_{i}\right) t^{2}+\left(c_{i} t^{\prime}+d_{i}\right) ;
$$

similarly, the osculant cubic of the $R^{4}$ at $t^{\prime}$ is

$$
x_{i}=\left(a_{i} t^{\prime}+b_{i}\right) t^{3}+3\left(b_{i} t^{\prime}+c_{i}\right) t^{2}+3\left(c_{i} t^{\prime}+d_{i}\right) t+\left(d_{i} t^{\prime}+e_{i}\right) ;
$$

and the osculant cubic of the $R^{5}$ at $t^{\prime}$ is

$$
\begin{aligned}
x_{i}=( & \left.a_{i} t^{2}+2 b_{i} t^{\prime}+c_{i}\right) t^{3}+3\left(b_{i} t^{\prime 2}+2 c_{i} t^{\prime}+d_{i}\right) t^{2} \\
& +3\left(c_{i} t^{2}+2 d_{i} t^{\prime}+e_{i}\right) t+\left(d_{i} t^{2}+2 e_{i} t^{\prime}+f_{i}\right) \quad(i=0,1,2) .
\end{aligned}
$$

If the equation of the covariant line of (15) is formed, analogous to (10), this equation will involve $t^{\prime}$ to the second power, and may be arranged as a quadratic in $t^{\prime}$. After these operations have been performed and $t^{\prime}$ changed to $t$, to indicate that it has become variable, the result may be written in the form

$$
[|a d x|-3|b c x|] t^{2}+[|a e x|-2|b d x|] t+[|b e x|-3|c d x|]=0 .
$$

This is a covariant conic of the $R^{4}$, and if (17) is compared with (3), its parametric equations may be seen to be

$$
\begin{aligned}
\xi_{i}=\left[\left(a_{\mu} d_{\nu}\right)-3\left(b_{\mu} c_{\nu}\right)\right] & t^{2}+\left[\left(a_{\mu} e_{\nu}\right)-2\left(b_{\mu} d_{\nu}\right)\right] t \\
+ & {\left[\left(b_{\mu} e_{\nu}\right)-3\left(c_{\mu} d_{\nu}\right)\right] \quad(i, \mu, \nu=0,1,2) . }
\end{aligned}
$$

It is readily seen that every $R^{n}$ of even degree has a covariant conic whose parametric equations may be found in the same way; this conic for any such $R^{n}$ of even degree is the envelope of the covariant lines of all osculant $(n-1)$-ics of the $R^{n}$.

If the equation of the covariant line of (16) is formed, the result will involve $t^{\prime}$ to the fourth power, and by a repetition of the process used above, the equations of the resulting covariant rational quartic in lines are

$$
\begin{aligned}
\xi_{i}= & {\left[\left(a_{\mu} d_{\nu}\right)-3\left(b_{\mu} c_{\nu}\right)\right] t^{4}+\left[2\left(a_{\mu} e_{\nu}\right)-4\left(b_{\mu} d_{\nu}\right)\right] t^{3} } \\
+ & {\left[\left(a_{\mu} f_{\nu}\right)+\left(b_{\mu} e_{\nu}\right)-8\left(c_{\mu} d_{\nu}\right)\right] t^{2}+\left[2\left(b_{\mu} f_{\nu}\right)-4\left(c_{\mu} e_{\nu}\right)\right] t } \\
& +\left[\left(c_{\mu} f_{\nu}\right)-3\left(d_{\mu} e_{\nu}\right)\right] \quad(i, \mu, \nu=0,1,2) .
\end{aligned}
$$

In the same way the $L_{n-2}$ of all osculant $(n-2)$-ics of $R^{n}$ of odd order gives rise to a covariant rational quartic of the $R^{n}$. I shall treat this curve (19) in a later section of this paper as a type of covariant rational quartic of an $R^{n}$ of odd order.

4. Relation Between Transvectants and Envelopes of Covariant Lines of Osculants. Equation (17) is exactly the same as (7) when $n=4$ and $r=3$. That is, the third transvectant of two line sections of the $R^{4}$ together with 
the translation scheme is the same as the envelope of covariant lines of all osculant cubics of the $R^{4}$. Similarly, the curve whose equations are given in (19) is the third transvectant of two line sections of the $R^{5}$, or may be obtained as the envelope of the covariant lines of all osculant cubics of the $R^{5}$. In general, if $n$ is even, the $(n-1)$ th transvectant of two line sections of the $R^{n}$ gives rise to a covariant conic of the $R^{n}$ which may be defined as the envelope of the covariant lines of all osculant $(n-1)$-ics of the $R^{n}$. In the same way the $(n-3)$ th transvectant of two line sections of the $R^{n}$ yields a covariant rational sextic of the $R^{n}$ which is identical with the envelope of covariant lines of all osculant $(n-3)$-ics of the $R^{n}$. More generally stated, the $(n-p)$ th transvectant of two line sections of the $R^{n}$, when $n$ is even, and $p$ odd, is the same as the envelope of covariant lines of all osculant $(n-p)$ ics of the $R^{n}$. When $n$ is odd the $(n-2)$ th transvectant of two line sections gives rise to a rational quartic in lines which is a covariant of the $R^{n}$, and which may be looked upon as the envelope of covariant lines of all osculant $(n-2)$-ics of the $R^{n}$; the envelope of covariant lines of all osculant $(n-q)$ ics is a rational curve in lines of degree $2 q$ of which the above quartic is a special case. A formal proof of these statements seems unnecessary as it would be evident to anyone who understands how to expand (7) and carry out the translation scheme suggested. I wish to consider in some detail the rational quartics mentioned above and in particular (19), which is the simplest of these with one exception, namely, if $n=3$ this curve becomes the tangential equation of the $R^{3}$ which has only one invariant. Also, the $R^{5}$ is the most interesting plane curve in this connection for a reason which will appear later; it is what may be called self-conjugate.

5. Invariants of $R^{n}$ Illustrated by the Particular Case of the $R^{5}$. An invariant of any $R^{n}$ given by equations (2) is expressible in terms of threerowed determinants of the type $|a b c|$, or determinants formed by replacing the $x$ 's of (8) by any other subscribed letter, say by $d$ 's which would give $|a b d|$. The invariants of (19) are invariants of the $R^{5}$; each three-rowed determinant of the matrix of coefficients of (19) is expressible as a quadratic function of the determinants of the type $|a b c|$. In my dissertation* I showed how certain combinants could be interpreted as invariants of the $R^{4}$. These combinants form the complete set of invariants of the $R^{4}$, and are expressible in terms of eight quantities $\alpha, \alpha^{\prime}, \beta, \beta^{\prime}, \mu, \mu^{\prime}, \lambda, \lambda^{\prime}, \gamma$, and $\delta$. I shall now give the values which must be assigned to these eight quantities so that they may be considered invariants of the $R^{5}$; these, of course, would not constitute the complete system of the $R^{5}$. Incidentally we may observe that the same process may be applied to find what values the greek letters must have so that these combinants mentioned may be interpreted as invariants of any $R^{n}$ of odd order.

*These Transactions, vol. 12 (1911), pp. 295-310. 
It is well known* that

$$
\left|\begin{array}{lll}
c_{1} f_{2}-c_{2} f_{1} & b_{1} e_{2}-b_{2} e_{1} & a_{1} d_{2}-a_{2} d_{1} \\
c_{2} f_{0}-c_{0} f_{2} & b_{2} e_{0}-b_{0} e_{2} & a_{2} d_{0}-a_{0} d_{2} \\
c_{0} f_{1}-c_{1} f_{0} & b_{0} e_{1}-b_{1} e_{0} & a_{0} d_{1}-a_{1} d_{0}
\end{array}\right|=|a b d||c e f|-|a d e||b c f|
$$

Using this as a basis of calculation the values of the greek letters which must be substituted in Salmon's combinants $\dagger$ to obtain invariants of the $R^{5}$ are

$$
\begin{aligned}
& \alpha=-16|c d f||b c f|+4|a c f||c e f|+4|c e f||b c e|-6|a b f||d e f| \\
& -2|b c f||b e f|-6|b d e||b e f|+48|b d e||c d f|-48|b c d||d e f| \\
& -12|c d e||a e f|-96|c d e|^{2} \text {. } \\
& \beta=2|a e f||a c f|-4|b d f||a c f|-2|a b e||c e f|-4|b c f||b d e| \\
& +16|c d f||a c e|-32|c d f||b c d|-6 \mid \text { aef }|| \text { ade }|+12| b d f|| a d e \mid \\
& -12 \mid \text { def }\left.|| a b d|-48| a d e|| c d e|+12| b d e\right|^{2} \text {. } \\
& \lambda=4|a e f||b c f|-12|a e f||b d e|-12 \mid \text { abe }|| \text { def }|+8| \text { ace }|| c e f \mid \\
& -8|b c f||b d f|+24|b d e||b d f|-16|c e f||b c d|-48|c d e||b d e| \text {. } \\
& \mu=2|a d f||b c f|-6|a b d||d e f|-6|a d f||b d e|+4|a c d||c e f| \\
& +12|a d e||c d e|-6|b c f|^{2}+18|b d e||b c f|-36|c d e||b c e| . \\
& \gamma=4|a b f||a e f|-8|a c e||a e f|-4|a b e||b e f|-32|a d e||b c f| \\
& +48 \mid \text { ace }|| b d f|-64| \text { ace }|| c d e|-8| a b f|| b d f|-16| a b d|| c e f \mid \\
& +16|b c e||b d e|-64|b d f||b c d|+128|b c d||c d e| \text {. } \\
& \delta=|a c f||a d f|-3|a d e||a d f|-|a b d||c e f|-3|a d e||b d e| \\
& +8|a c d||c d f|-3|a c f||b c f|+10|a d e||b c f|-9|a b c||d e f| \\
& -3|b c f||b c e|+9|b c e||b d e|-72|c d e||b c d| \text {. }
\end{aligned}
$$

The values of $\alpha^{\prime}, \beta^{\prime}, \lambda^{\prime}$, and $\mu^{\prime}$ are not written out because they may be obtained by making the following interchanges in the values of the corresponding unprimed letters:

$$
a \text { and } f ; \quad b \text { and } e \text {; and } c \text { and } d .
$$

I have already explained what is meant by the self-conjugate property of the plane $R^{5}$ in a previous paper. It will be sufficient here with this reference

* Muir, The Theory of Determinants, pp. 142-143.

$\dagger$ Higher Algebra, third edition, pp. 200-206.

Trans. Am. Math. Soc. 4 
to recall that every invariant of the $R^{5}$ may be transformed either into itself or into another invariant of the $R^{5}$ by the substitution of complementary determinants and allowance for numerical coefficients. That is, if in Salmon's combinants $A, B, C$, and $D$ the above values are substituted for the greek letters we have four invariants of the $R^{5}$; if in these invariants we substitute, for instance, $50|c e f|$ for $|a b d|$, and $5|a e f|$ for $|b c d|$ and the other similar complementary determinants multiplied by a proper coefficient, we obtain four other invariants of the $R^{5}$. If the same operations are again performed multiples of the original invariants $A, B, C$, and $D$ of the $R^{5}$ are formed. It is fairly evident upon reflection that this transformation is necessarily involutory. These eight invariants together with the cusp* and undulation invariants may be used as the basis of a study of invariants of the $R^{5}$; relations among them will be reserved for a later discussion.

Pennsyluania State College,

December, 1914.

*Annals of Mathematics, vol. 14 (1913), pp. 203-205. 